\title{
A PROBLEM OF PAUL ERdÖS ON GROUPS
}

\author{
Dedicated to George Szekeres for his 65th birthday
}

B. H. NEUMANN

(Received 24 January 1975)

Communicated by Jennifer Seberry Wallis

\section{Introduction}

Let $G$ be a group, and associate with $G$ a graph ${ }^{1} \Gamma=\Gamma(G)$ as follows: the vertices of $\Gamma$ are the elements of $G$, and two vertices $g, h$ of $\Gamma$ are joined by an undirected edge if, and only if, $g$ and $h$ do not commute as elements of $G$, that is $[g, h] \neq 1$ [where $[g, h]$ is the commutator $g^{-1} h^{-1} g h$, and 1 is the unit element of the groups that occur as well as the integer, according to context]. We are interested in complete subgraphs of $\Gamma$, or equivalently in sets of elements of $G$ no two of which commute.

Paul Erdös recently ${ }^{2}$ posed the following problem:

Let $G$ be such that $\Gamma$ contains no infinite complete subgraph; is there then a finite bound on the cardinality of complete subgraphs of $\Gamma$ ?

The purpose of this note is to answer this question affirmatively. It turns out, in fact, that the class of groups whose graph contains no infinite complete subgraph - let us call them Paul Erdös groups, or PE-groups for short coincides with the class of groups whose centre has finite index; these latter groups are called FIZ-groups for short, and have been studied in other contexts; see, for example, Neumann (1955) and the literature there quoted, or Chapter 4 of Robinson's book (1972), where FIZ-groups are called central-by-finite.

1 There are other, classical - and group-theoretically more significant - ways of associating a graph with a group. We here restrict attention to the graphs $\Gamma$, and "the graph of $G$ " will mean $\Gamma(G)$.

2 At the 15th Summer Research Institute of the Australian Mathematical Society, at the University of New South Wales, 13 January-14 February 1975. 


\section{Preliminaries}

We make the convention that [PE] stands for the class of PE-groups, [FIZ] for the class of FIZ-groups, and similarly for other group-theoretical properties. In particular we denote by [FC] the class of groups in which all classes of conjugate elements are finite. It is a well-known fact, and indeed almost trivial, that all FIZ-groups are FC-groups. Thus the following lemma is a step in the right direction.

\section{Lemma 1. All PE-groups are FC-groups.}

Proof. Let $G$ be a group not in [FC]; we show that $G$ is not in [PE] either. Let $g \in G$ be an element with an infinite class of conjugates, and let $T$ be an infinite set of elements of $G$ such that distinct elements of $T$ produce distinct conjugates of $g$ : thus if $s, t \in T$ and $s \neq t$, then $s^{-1} g s \neq t^{-1} g t$. Consider the restriction $\Gamma(T)$ of $\Gamma(G)$ to $T$. By Ramsey's Theorem $\Gamma(T)$ either contains an infinite complete subgraph - in which case $G \notin[P E]$ and we have finished - or an infinite independent subset $U$, that is an infinite set of vertices without any edges. The elements of $U$, as elements of $G$, commute with each other. Now consider the set

$$
g U=\{g u \mid u \in U\} .
$$

If $u, v$ are distinct elements of $U$, then the commutator

$$
\begin{aligned}
{[g u, g v] } & =(g u)^{-1}(g v)^{-1} g u g v \\
& =u^{-1} g^{-1} v^{-1} u g v \\
& =u^{-1} g^{-1} u v^{-1} g v \neq 1,
\end{aligned}
$$

as $u^{-1} g u \neq v^{-1} g v$, by the choice of $T$. Thus no two distinct elements of $g U$ commute, $\Gamma(g U)$ is an infinite complete graph, and $G \notin[P E]$; and the lemma follows.

We denote, as usual, the centralizer of an element $g \in G$ by $C_{G}(g)$, and the centralizer of a set $S$ of elements of $G$ by $C_{G}(S)$; thus

$$
C_{G}(S)=\bigcap_{s \in S} C_{G}(s)
$$

The index $\left|G: C_{G}(g)\right|$ of the centralizer of $g \in G$ equals the cardinal of the class of conjugates of $g$. Thus $G \in[F C]$ if, and only if, $\left|G: C_{G}(g)\right|$ is finite for all $g \in G$.

The reader is reminded that the intersection of a finite set of subgroups of a group $G$ has finite index in $G$ if each of the subgroups in the set has finite index in $G$. Thus $G \in[F C]$ if, and only if, $\left|G: C_{G}(S)\right|$ is finite for every finite set $S$ of elements of $G$. 
We also remind the reader that if the set $S$ of elements of $G$ generates $G$, then $C_{G}(S)=Z(G)$, the centre of $G$. It follows at once that-as is well known - every finitely generated FC-group is a FIZ-group.

Lemma 2. Let $G \in[F C]$ have an abelian subgroup $A$ of finite index. Then $G \in[F I Z]$.

Proof. We can generate $G$ by a set $Q$, say, of generators of $A$ and a finite set $R$, say, of further generators, for example one element out of each (right) coset of $A$ in $G$. Put $S=Q \cup R$. The centre of $G$ then is

$$
Z(G)=C_{G}(S)=C_{G}(Q) \cap C_{G}(R) .
$$

Now $Q$ lies in the abelian subgroup $A$, hence $A \subseteq C_{G}(Q)$, and $C_{G}(Q)$ has finite index in $G$. But so has $C_{G}(R)$, as $R$ is finite and $G \in[\mathrm{FC}]$. Thus also $Z(G)$ has finite index in $G$, and the lemma follows.

The converse of the lemma, namely that if $G \in$ [FIZ], then $G$ has an abelian subgroup of finite index, is trivial. More important for us is a negative reformulation of the lemma.

Corollary 3. Let $G \in[F C]-[F I Z]$ have a subgroup $A$ of finite index. Then $A$ is not abelian.

\section{The main result}

As we already know that both PE-groups and FIZ-groups are FC-groups, we now have to show that a group $G \in[\mathrm{FC}]-[\mathrm{FIZ}]$ cannot be a PE-group. The rest of the characterization of [PE] will be easy.

Lemma 4. Let $G \in[F C]-[F I Z]$, and assume that $G$ contains two finite sequences of $n$ elements

$$
\left(a_{1}, a_{2}, \cdots, a_{n}\right), \quad\left(b_{1}, b_{2}, \cdots, b_{n}\right)
$$

with the following properties:

(i) if $i \neq j$, then $\left[a_{i}, a_{j}\right] \neq 1$;

(ii) if $i \neq j$, then $\left[a_{i}, b_{i}\right]=1$;

(iii) for all $i,\left[a_{i}, b_{i}\right] \neq 1$;

(iv) for all $i, j,\left[b_{i}, b_{i}\right]=1$.

Then $G$ contains two further elements $a_{n+1}, b_{n+1}$ such that (i), (ii), (iii), (iv) remain valid for the sequences

$$
\left(a_{1}, a_{2}, \cdots a_{n+1}\right), \quad\left(b_{1}, b_{2}, \cdots, b_{n+1}\right)
$$

of length $n+1$. 
Before we embark on the proof, we pause to look at the restriction of the graph $\Gamma(G)$ to the set of elements $\left\{a_{1}, a_{2}, \cdots, a_{n}, b_{1}, b_{2}, \cdots, b_{n}\right\}$ : we have a complete subgraph with vertices $a_{1}, a_{2}, \cdots, a_{n}$, and each $a_{i}$ is joined to the vertex $b_{i}$, and there are no other edges.

Proof of Lemma 4. Put

$$
\left.A=C_{G}\left(a_{1}, a_{2}, \cdots, a_{n}, b_{1}, b_{2}, \cdots, b_{n}\right\}\right) .
$$

Then $A$ has finite index in $G$, and as $G \in[\mathrm{FC}]-[\mathrm{FIZ}]$ by assumption, $A$ is, by Corollary 3 , not abelian. Thus we may pick two elements from $A$, say $a$ and $b$, such that $[a, b] \neq 1$. Now put

$$
\begin{aligned}
a_{n+1} & =a \cdot b_{1} b_{2} \cdot \cdots \cdot b_{n}, \\
b_{n+1} & =b .
\end{aligned}
$$

Then, for $1 \leqq i \leqq n$,

$$
\left[a_{i}, a_{n+1}\right]=\left[a_{i}, b_{i}\right] \neq 1,
$$

as $a$ and all $b_{j}$ other than $b_{i}$ commute with $a_{i}$;

$$
\left[a_{n+1}, b_{i}\right]=\left[a_{i}, b_{n+1}\right]=1,
$$

as $a$ and all $b_{i}$ commute with $b_{i}$ and $a_{i}$ commutes with $b$;

$$
\left[a_{n+1}, b_{n+1}\right] \neq 1,
$$

as $a$ and $b=b_{n+1}$ do not commute, but $b$ and all $b_{i}$ do;

$$
\left[b_{i}, b_{n+1}\right]=1 \text {, }
$$

obviously. The lemma thus follows.

Corollary 5. If $G \in[F C]-[F I Z]$, then $G \notin[P E]$.

We only have to use Lemma 4 to build up, inductively, an infinite sequence $\left(a_{1}, a_{2}, a_{3}, \cdots\right)$ of pairwise non-commuting elements, starting from a pair $a_{1}, b_{1}$ of non-commuting elements, as $G$ is obviously not abelian. This is now, but for the almost trivial converse, our main result.

THEOREM 6. The group G is a PE-group if, and only if, it is a FIZ-group.

Proof. We have already seen that every PE-group must be a FIZ-group: this follows from Lemma 1 and Corollary 5. However, it is obvious that every FIZ-group is a PE-group; for if $G \in[\mathrm{FIZ}]$ and $|G: Z(G)|=n$, then every set of $n+1$ elements of $G$ must contain two that are congruent modulo the centre $Z(G)$, and thus commute. The theorem thus follows. 


\section{Odds and ends}

We have seen that if the centre of the group $G$ has index $n$ in $G$, then the graph $\Gamma(G)$ contains no complete subgraph of order greater than $n$; and this can be immediately improved to $n-1$ in general, and a little further if the arithmetical structure of $n$ is taken into account. Thus $n-1=3$ is attained for the quaternion group and also for the dihedral group of order 8 [these two groups have isomorphic graphs $\Gamma$ ]; and I owe to Dr M. F. Newman the remark that when $n=2^{d}$, then $n-1$ is attained by the free group of rank $d$ of the variety generated by the quaternion group; but for the symmetric group of degree 3 the index of the centre is $n=6$, while the biggest complete subgraph has order 4 . This immediately raises the question of a bound for the index of the centre, $|G: Z(G)|=n$, given that the graph $\Gamma(G)$ contains no complete subgraph of order greater than $\mathrm{m}$. One can obtain such a bound from the proof of Theorem 6 , namely

$$
\log n=O\left(m^{2}\right)
$$

but this bound is very crude, and my guess, based on no evidence, is

$$
n=O\left(m^{2}\right) \text {. }
$$

A similar question might be asked with respect to Lemma 2: if the FC-group $G$ has an abelian subgroup $A$, what estimate in terms of $|G: A|$ can be given for the index $n$ of the centre of $G$ ? The answer is: none. On only needs to take $A$ as an abelian group of finite odd order and make $G$ the splitting extension of $A$ by a cyclic group of order 2 whose generator inverts all elements of $A$. Then $|G: A|=2$, but the centre of $G$ is trivial, hence $n=|G|$, and this can be made as large as you please.

If $m$ is taken as the precise maximum order of a complete subgraph of $\Gamma(G)$, then not all integers occur as values of $m$. Paul Erdös remarked that 2 does not occur, for to any two elements $a, b$ that do not commute with each other there is a third, namely $a b$, that commutes with neither of them. It is then natural to ask what (finite) values of $m$ are possible. The answer is that 2 is the only exception. The dihedral group of order $4(m-1)$ is an example of a group whose graph contains a complete subgraph of order $m$, but none of greater order. The (easy) verification is omitted.

\section{References}

B. H. Neumann (1955), 'Groups with finite classes of conjugate subgroups', Math. Z. 63, 76-96. Derek J. S. Robinson (1972), Finiteness conditions and generalized soluble groups, Part 1 (Ergebnisse der Mathematik und ihrer Grenzgebiete, Band 62. Springer-Verlag, Berlin, Heidelberg, New York 1972). 
The Australian National University

and

Commonwealth Scientific and Industrial Research Organization,

Division of Mathematics and Statistics,

Canberra, Australia

Added 18 July 1975. I have just heard that the results in this paper were obtained also, by much the same methods, by Professor Ralph N. McKenzie, two or three months before I had them. He and Dr. Vance Faber hope to publish in due course some extensions of these results to higher cardinal numbers and to cancellation semigroups. 